\title{
PREDICTION $n$ UNITS OF TIME AHEAD ${ }^{1}$
}

\author{
TAKAHIKO NAKAZI AND KATUTOSHI TAKAHASHI
}

ABstract. The purpose of this note is to give a simple expression in terms of $w$ of the quantities

$$
\rho_{n}(w)=\inf _{f} \int_{0}^{2 \pi}\left|1+e^{i n \theta} f\right|^{2} w d \theta / 2 \pi \quad(n=0,1,2, \ldots),
$$

where $f$ ranges over the analytic trigonometric polynomials with mean value zero and $w$ is nonnegative and summable on the circle.

Helson [2, pp. 21-22] said that it is unreasonable to expect to have a simple expression in terms of $w$ for the quantities $\rho_{n}$ except $n=0$. $\rho_{0}(w)=$ $\exp \int_{0}^{2 \pi} \log w d \theta / 2 \pi$ is the famous Szegö theorem. We may assume $\log w$ is summable, because otherwise $\rho_{n}(w)=0$ for all $n$.

TheOREM. Let $w$ be nonnegative and summable on the circle. Suppose $\log w$ is summable and

$$
\log w(\theta) \sim \sum_{j=-\infty}^{\infty} a_{j} e^{i j \theta}
$$

Then

$$
\begin{aligned}
\rho_{n}(w) & =\inf _{f} \int_{0}^{2 \pi}\left|1+e^{i n \theta} f\right|^{2} w d \theta / 2 \pi \\
& =e^{a_{0}} \times \sum_{j=0}^{n}\left|\sum \frac{a_{1}^{m_{1}} a_{2}^{m_{2}} \cdots a_{n}^{m_{n}}}{m_{1} ! m_{2} ! \cdots m_{n} !}\right|^{2}
\end{aligned}
$$

where $n>1$ and $f$ ranges over the analytic trigonometric polynomials with mean value zero and $\Sigma^{\prime}$ is the summation of all permutations of nonnegative integers $m_{1}$, $m_{2}, \ldots, m_{n}$ with $m_{1}+2 m_{2}+\cdots+n m_{n}=j$ for each $j$.

PROOF. Set $g_{1}=\sum_{l=1}^{n} a_{l} z^{l}$ and $g_{2}=a_{0} / 2+\sum_{l>n+1}^{\infty} a_{n} z^{n}$, then their radial limits satisfy $w(\theta)=\left|\exp g_{1}\left(e^{i \theta}\right)\right|^{2}\left|\exp g_{2}\left(e^{i \theta}\right)\right|^{2}$ a.e. $\theta$. $\exp \left(g_{1}+g_{2}\right)$ is an outer function [3, p. 61] and so $\exp g_{2}$ is outer. Hence, if we note that there exist positive numbers $\varepsilon$ and $M$ with $0<\varepsilon<\left|\exp g_{1}\left(e^{i \theta}\right)\right|^{2}<M<\infty$, as in the proof of Szegö's theorem

Received by the editors July 9, 1979 and, in revised form, September 11, 1979 and November 9, 1979. AMS (MOS) subject classifications (1970). Primary 60G25, 30A98, 46J15.

Key words and phrases. Prediction theory, Szegö's theorem, $n$ units of time ahead, outer function, Fourier coefficients.

${ }^{1}$ Research supported in part from Grant-in-Aid Encouragement of Young Scientists. 


$$
\begin{aligned}
\rho_{n}(w) & =\inf _{f} \int\left|1+e^{i n \theta} f\right|^{2}\left|\exp g_{2}\right|^{2}\left|\exp g_{1}\right|^{2} d \theta / 2 \pi \\
& =\inf _{f} \int\left|\exp g_{2}+e^{i n \theta} f \exp g_{2}\right|^{2}\left|\exp g_{1}\right|^{2} d \theta / 2 \pi \\
& =\inf _{f} \int\left|e^{a_{0} / 2}+e^{i n \theta} f\right|^{2}\left|\exp g_{1}\right|^{2} d \theta / 2 \pi \\
& =e^{a_{0}} \inf _{f} \int\left|1+e^{i n \theta} f\right|^{2}\left|\exp g_{1}\right|^{2} d \theta / 2 \pi
\end{aligned}
$$

Since $\exp g_{1}$ is an outer function, if the Fourier coefficients of $\exp g_{1}$ are $\left\{b_{j}\right\}$, then (cf. [1, pp. 184-187], [2, p. 22])

$$
\begin{aligned}
\inf _{f} \int\left|1+e^{i n \theta} f\right|^{2}\left|\exp g_{1}\right|^{2} d \theta / 2 \pi \\
\quad=\sum_{j=0}^{n}\left|b_{j}\right|^{2} \exp g_{1}(z)=\prod_{l=1}^{n} \exp \left(a_{l} z^{l}\right)=\sum \frac{\left(a_{1} z\right)^{m_{1}} \cdots\left(a_{n} z^{n}\right)^{m_{n}}}{m_{1} ! \cdots m_{n} !}
\end{aligned}
$$

where the $m_{j}$ range independently over nonnegative integers. This implies the theorem.

The theorem shows the following:

$$
\begin{aligned}
\rho_{1}(w) & =\inf _{f} \int_{0}^{2 \pi}\left|1+e^{i \theta} f\right|^{2} w d \theta / 2 \pi \\
& =\exp \int_{0}^{2} \log w d \theta / 2 \pi\left\{1+\left|\int_{0}^{2 \pi}(\log w) e^{i \theta} d \theta / 2 \pi\right|^{2}\right\} .
\end{aligned}
$$

If $w$ is nonnegative and summable, and $\log w$ is summable, it is known (cf. [2, p. 20]) that $w=|g|^{2}$ for some outer $g$. The theorem gives a simple expression in terms of $w$ of the Fourier coefficients of outer function $g$ (cf. [4]).

We wish to express our appreciation to the referee for several valuable suggestions.

\section{REFERENCES}

1. U. Grenander and G. Szegö, Toeplitz forms and their applications, Univ. of California Press, Berkeley and Los Angeles, 1958.

2. H. Helson, Lectures on invariant subspaces, Academic Press, New York, 1964.

3. K. Hoffman, Banach spaces of analytic functions, Prentice-Hall, Englewood Cliffs, N.J., 1962.

4. T. L. Kriete III, On the Fourier coefficients of outer functions, Indiana Univ. Math. J. 20 (1970), 147-155.

Division of Applied Mathematics, Research Institute of Applied Elactrictiy, Hoxkaido UNIVERSTTY, SAPPORO, JAPAN 